\title{
NOTA
}

\section{COMPOSICIÓN Y ABUNDANCIA DE LA ARTROPODOFAUNA TERRESTRE EN LA PENÍNSULA FILDES}

\author{
Mariana C. Trillo ${ }^{1,2}$ *, Estefanía Stanley² , Valeria Rodríguez ${ }^{1}$, Álvaro Laborda ${ }^{1}$, Manuel Castro ${ }^{1}$ \\ 1 Sección Entomología. Facultad de Ciencias, Universidad de la República, Montevideo, Uruguay. \\ 2 Departamento de Ecología y Biología Evolutiva. Instituto de Investigaciones Biológicas Clemente Estable, \\ Montevideo, Uruguay.
}

* Autor para correspondencia: Mariana Trillo - maritrillo87@gmail.com

\section{RESUMEN}

Se llevó a cabo el primer relevamiento completo de la artropodofauna tesrrestre de la Península Fildes, cercana a la Base Científica Artigas. Los muestreos se realizaron durante febrero de 2015 en el marco del proyecto "Composición y estructura de la Artrópodo-fauna en áreas cercanas a la Base Científica Antártica Artigas" financiado por el Instituto Antártico Uruguayo (IAU). Se encontraron cuatro órdenes de artrópodos, incluyendo Coleoptera, que resulta el primer registro del orden para las Shettland del Sur.

Palabras clave: Antártida, Artrópodos, Bioindicadores, Coleóptera

\section{ABSTRACT}

Diversity and abundance of terrestrial arthropodofauna in the Artigas Antarctic Scientific Base (BCAA). The first complete survey of the terrestrial and aerial artopodofauna present in the Artigas Scientific Base and its surroundings was carried out. The samplings were performed during February 2015 within the project "Composición y estructura de la Artrópodo-fauna en áreas cercanas a la Base Científica Antártica Artigas" founded by Instituto Antártico Uruguayo (IAU). We found four arthropod orders, in which Coleoptera is the first report of the order for the South Shetland Islands.

Keywords: Antarctica, Arthropods, Bioindicators, Coleoptera

Los artrópodos constituyen parte fundamental de los ecosistemas terrestres, ocupando gran diversidad de nichos funcionales (Seastedt \& Crossley, 1984). Responden rápidamente a cambios ambientales por lo que se los considera indicadores muy efectivos para monitorear y/o planificar estrategias de manejo en áreas naturales (Longcore, 2003; Uehara-Prado et al., 2009). El continente Antártico constituye un área natural poco perturbada, con ecosistemas de características únicas (Kennedy, 1995; Convey \& Stevens, 2007). Los ecosistemas antárticos son particularmente frágiles ante los disturbios, poseen una muy baja resiliencia y están siendo afectados por el rápido incremento en la actividad humana (Frenot et al., 2005; Hughes \& Convey, 2012).

Las Islas Shetland del Sur se caracterizan por tener variaciones estacionales y un clima marítimo frío (Serrano, 2003). Durante el verano, las zonas costeras quedan libres de hielo y permiten el desarrollo de importantes comunidades vegetales (Kim et al., 2007). Estas áreas de superficie reducida, concentran la mayor biodiversidad terrestre antártica (Tilbrook, 1967).

La Península Fildes está localizada en la Isla Rey Jorge la cual forma parte del mencionado archipiélago. La península ha sido categorizada como Área Antártica Especialmente Protegida (ASPA) (Patrimonio Natural, 2009) y en ella se encuentra la Base Científica Antártica Artigas (BCAA) (62010' S 5850' O) desde el año 1984, siendo Uruguay miembro del tratado Antártico desde el año 1985 con fines de investigación científica (Rothwell, 1990). Debido a los pocos estudios realizados sobre artrópodos en las inmediaciones de la BCAA hasta la fecha (Convey et al., 2008; Volonterio et al., 2013), se torna fundamental conocer en profundidad la fauna de artrópodos presente en la región para evaluar los posibles disturbios en el continente.

El objetivo de este trabajo fue realizar un relevamiento de la artropodofauna terrestre en las inmediaciones de la Base Científica Antártica Artigas (Fig. 1) y de los tanques de combustible rusos (62011'35.62"S 58056'1.56"O), así como en la isla Ardley (62012'12.38"S 58056'56.10"O), en el marco del proyecto Composición y estructura de la Artrópodofauna en áreas cercanas a la Base Científica Antártica Artigas del Instituto Antártico Uruguayo (IAU).

Los sitios fueron seleccionados de acuerdo a la logística propuesta por los Coordinadores Científicos del Instituto Antártico Uruguayo, considerando su accesibilidad y tratando de abarcar la mayor diversidad de hábitats posible.

Para ello, un recolector tomó muestras entre el 13 y el 19 de febrero de 2015 utilizando cuatro métodos diferentes de recolección de artrópodos: trampas de caída, trampas de intercepción de vuelo, embudo de Berlese y recolección manual. Las trampas de caída consistieron en recipientes plásticos de $95 \mathrm{~mm}$ de diámetro y $110 \mathrm{~mm}$ de profundidad con un techo 


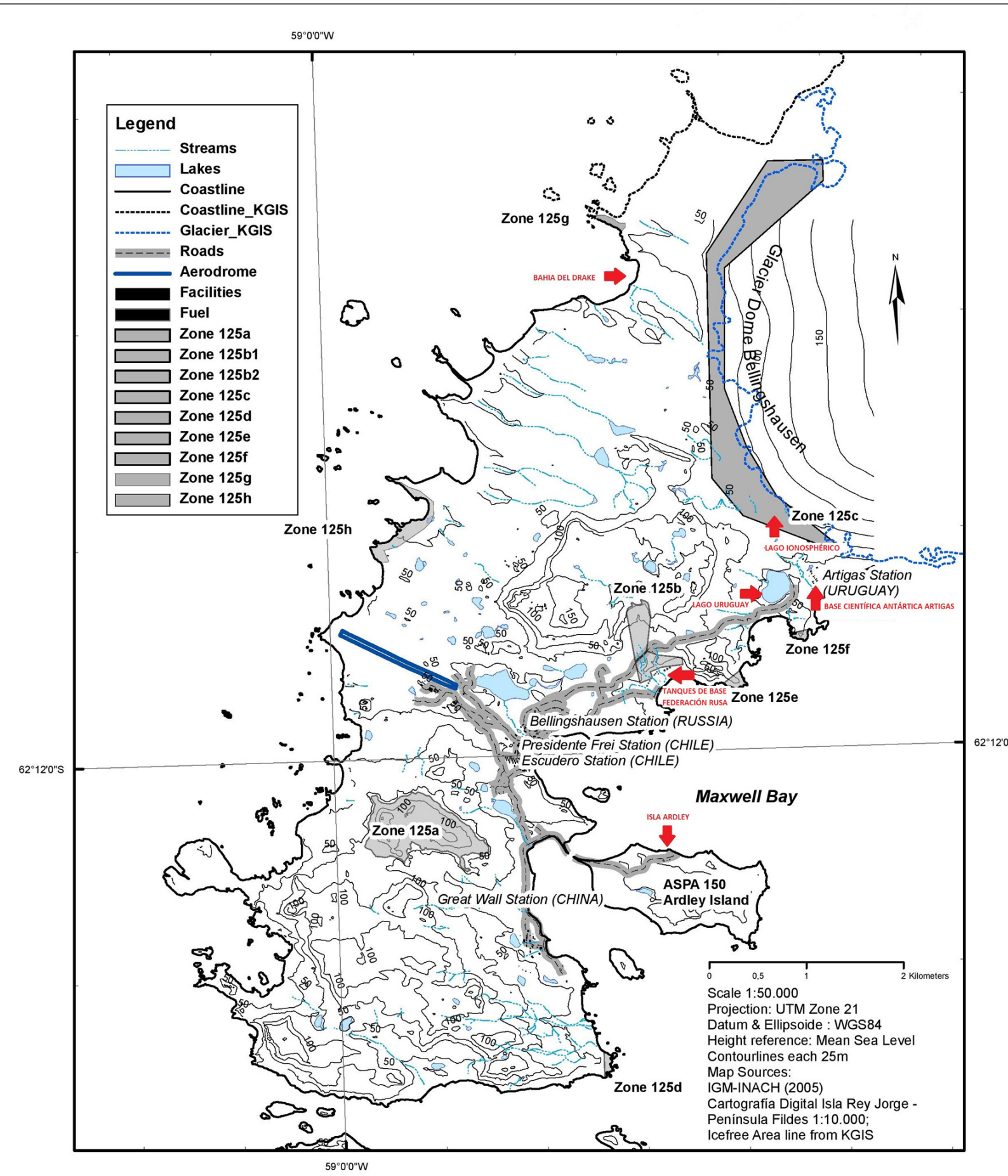

Fig. 1. Mapa de la Península Fildes. Los sitios donde se realizaron muestreos se señalan con flechas rojas: Base Científica Antártica Artigas (BCAA), Isla Ardley, Lago lonosférico, Lago Uruguay, Pasaje Drake y Tanques de Base Federación Rusa.

plástico para evitar el ingreso del agua de lluvia (Fig. 2a). Cada trampa contenía formol al $10 \%$ como líquido conservador y detergente para prevenir el escape de los ejemplares. Las mismas fueron colocadas en dos transectas de 10 trampas separadas un metro entre sí, dispuestas una en paralelo y otra transversal a la orilla de los lagos Uruguay (62011'08.3"S 58054'54.5”O) e lonosférico (62010'51.90"S 58054'37.70”O) (Fig. 1). Las trampas de intercepción de vuelo consistieron en 4 láminas de $20 \times 20 \mathrm{~cm}$, con una de sus caras cubierta por una sustancia adherente. Las láminas se dispusieron en forma perpendicular al suelo en 
Tabla 1. Órdenes de artrópodos y número total de ejemplares recolectados en los diferentes puntos de muestreo.

\begin{tabular}{lcccccc} 
& BCAA & $\begin{array}{c}\text { Isla } \\
\text { Ardley }\end{array}$ & $\begin{array}{c}\text { Lago } \\
\text { Ionosférico }\end{array}$ & $\begin{array}{c}\text { Lago } \\
\text { Uruguay }\end{array}$ & $\begin{array}{c}\text { Pasaje } \\
\text { Drake }\end{array}$ & $\begin{array}{c}\text { Tanques de Base } \\
\text { Federación Rusa }\end{array}$ \\
\hline Acaridae & 0 & 3765 & 0 & 4 & 5 & 4 \\
Collembola & 37 & 879 & 0 & 100 & 92 & 1857 \\
Coleoptera & 0 & 0 & 0 & 1 & 0 & 0 \\
Diptera & 32 & 0 & 533 & 9299 & 0 & 0 \\
\hline
\end{tabular}

soportes a un metro de altura, cada una orientada hacia uno de los puntos cardinales. Se colocaron dos de estas trampas, una fuera de la Base Científica Antártica Artigas (BCAA) (62011'05.1"S 5854'15.4"O) próximo al módulo del comedor y otra en el Lago Uruguay a una distancia de $20 \mathrm{~m}$ de la orilla, dejándolas actuar por 24 horas. Se realizó una recolección manual ad libitum en las 10 cámaras sépticas de la BCAA (Fig. 2b), en el módulo de radio (estación de comunicaciones de radio) y en la cámara de frío y alimentos de la cocina. Por último, en la Isla Ardley y zonas aledañas al Pasaje Drake BCAA, Lago Uruguay y los tanques de la Base Rusa, se tomaron 3 muestras de suelo en cada sitio, que fueron procesadas mediante el embudo de Berlese (Fig. 3). Los ejemplares recolectados se preservaron en alcohol $75 \%$ y fueron depositados en la Colección Entomológica de la Facultad de Ciencias, Uruguay.

Se obtuvieron un total de 16.608 ejemplares pertenecientes a los siguientes órdenes: Acari, Coleoptera, Collembola y Diptera (Tabla 1). Con el método de trampas de caída realizado en la zona de los lagos, se obtuvieron 9829 individuos (1 Acaridae,
6 Collembola y 9822 Diptera), en su gran mayoría individuos de la especie Parochlus steinenii (Gercke, 1889) (Diptera, Chironomidae) (4451 hembras, 5360 machos y diez parejas en cópula) (Fig.4a). De éstos, el $95 \%$ fueron recolectados del Lago Uruguay y el $5 \%$ en el Lago del Ionosférico. La baja abundancia de $P$. steinenii en el Lago del lonosférico podría atribuirse a la ausencia de vegetación e influencia directa del glaciar (Hahn \& Reinhardt, 2006) ya que la dieta de los estadios juveniles son los líquenes acuáticos (Convey \& Block, 1996). En la línea de trampas transversales, se observó un incremento de la abundancia de esta especie a medida que disminuye la distancia a la orilla del lago, mientras que en las líneas dispuestas en paralelo a la orilla se obtuvieron abundancias similares en todas las trampas. Los adultos $P$. steinenii se observaron agrupados en las orillas de los lagos, esta ubicación les brindaría protección del viento facilitando el apareamiento entre los ambos sexos. Las larvas son acuáticas y las pupas en su último estadio flotan usando el viento como medio para llegar a la orilla donde posteriormente emergen a individuos adultos (Barticevic, 2015).

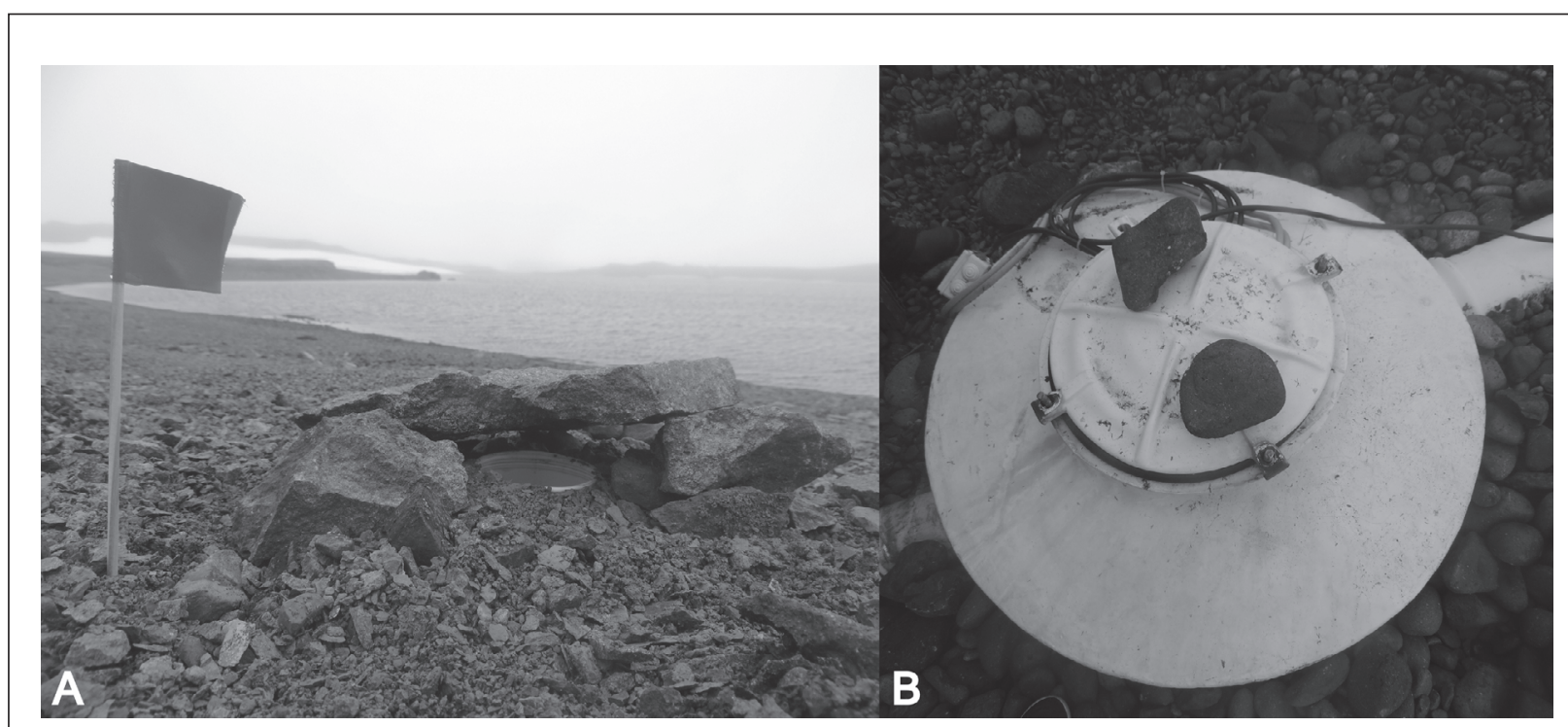

Fig. 2. A) Trampas de caída. B) Cámaras sépticas donde se realizó la recolección manual. 


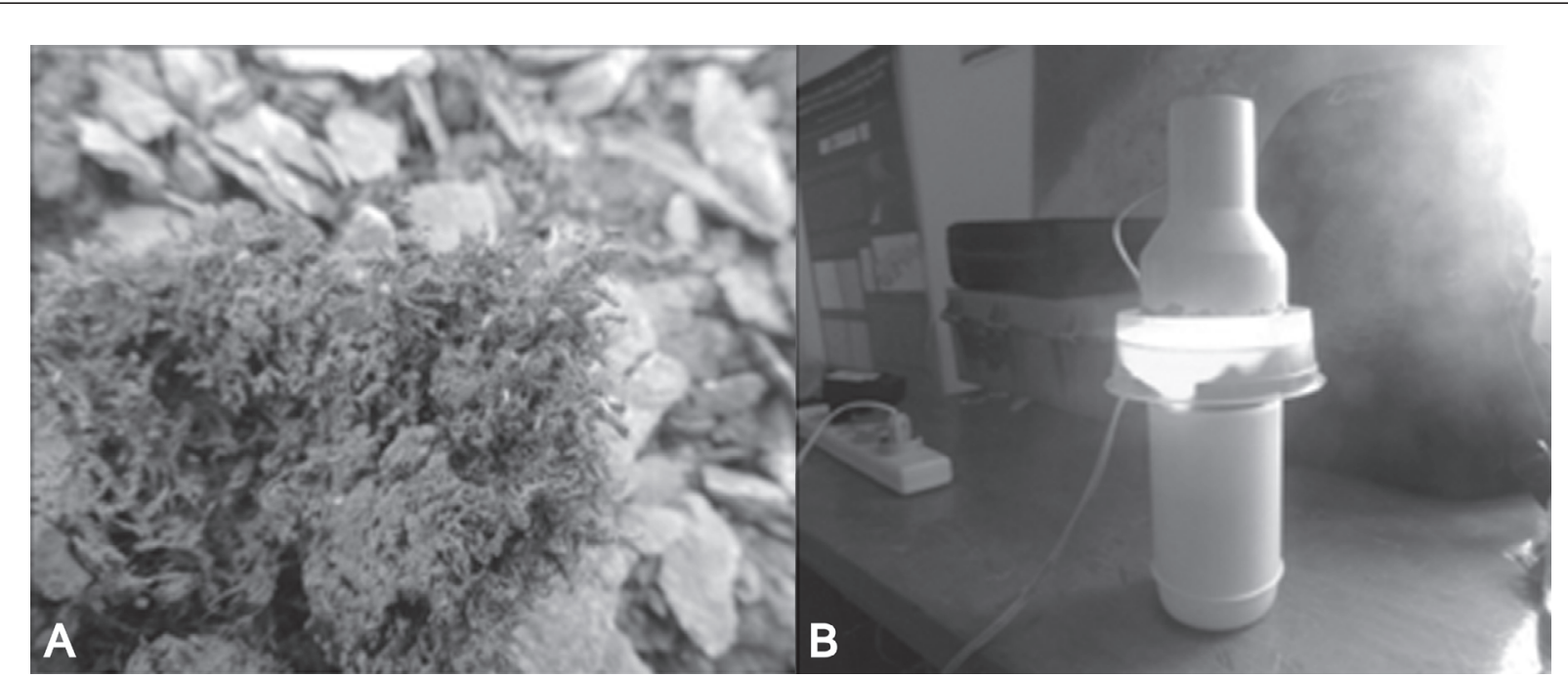

Fig. 3. A) Colecta de materia orgánica. B) Técnica embudo de Berlese.

Las trampas de intercepción de vuelo no recolectaron ejemplares, lo que puede deberse a que en este ambiente los insectos alados como $P$. steinenii vuelan a alturas menores al metro para evitar el viento (Gañán Mora et al., 2015). Cabe resaltar que es la primera vez que se utiliza este método de muestreo en la Isla Rey Jorge. En virtud de los resultados, futuros estudios se enfocarán en colocar trampas a alturas más bajas.

Mediante la metodología de recolección manual en las cámaras sépticas, la BCAA y el módulo de radio la única especie registrada fue Trichocera maculipennis Meigen, 1818 (Diptera, Trichoceridae) (Fig.4b), previamente reportada por Volonterio et al. (2013). Se trata de una especie exótica asociada a la urbanización y posiblemente transportada al continente antártico junto con los caños utilizados para construir las cámaras sépticas. Estos depósitos oficiarían de incubadoras para su desarrollo, debido a que las aguas residuales de dichas cámaras no se congelan gracias al vertido de anticongelantes, se encuentran a resguardo del viento y mantienen una temperatura más elevada con respecto al exterior. Se recolectaron un total de 30 individuos, 13 hembras y 15 machos incluyendo una pareja en cópula. Los individuos fueron encontrados dentro del área de la BCAA: en las cámaras sépticas de los módulos habitacionales (50\%), en la Estación de comunicaciones de radio (4\%) y en la cámara de frío de alimentos de la cocina (46\%). Un ejemplar se encontró al aire libre, a 15 metros de las cámaras y la pareja en cópula en la cámara de frío de la

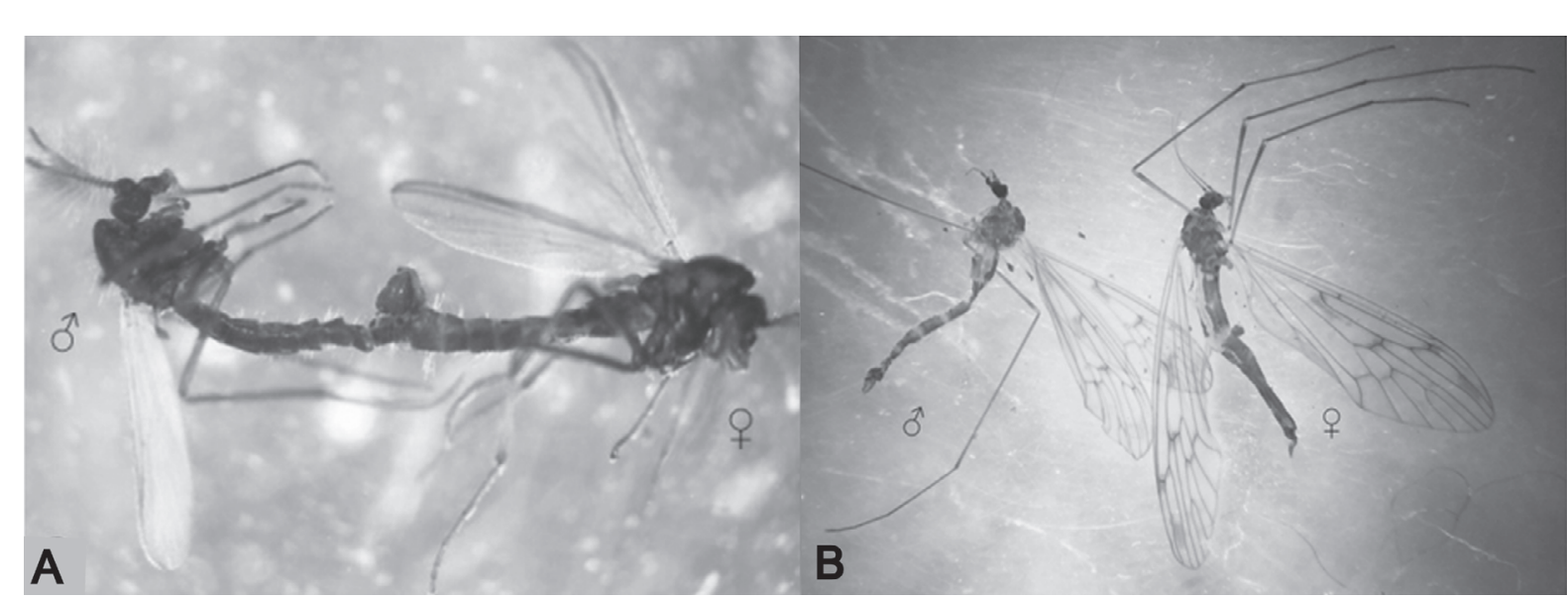

Fig. 4. Especies de dípteros colectados en los muestreos por trampa de caída. A) pareja en cópula Parochlus steinenii; B) macho y hembra de Trichocera maculipennis. 


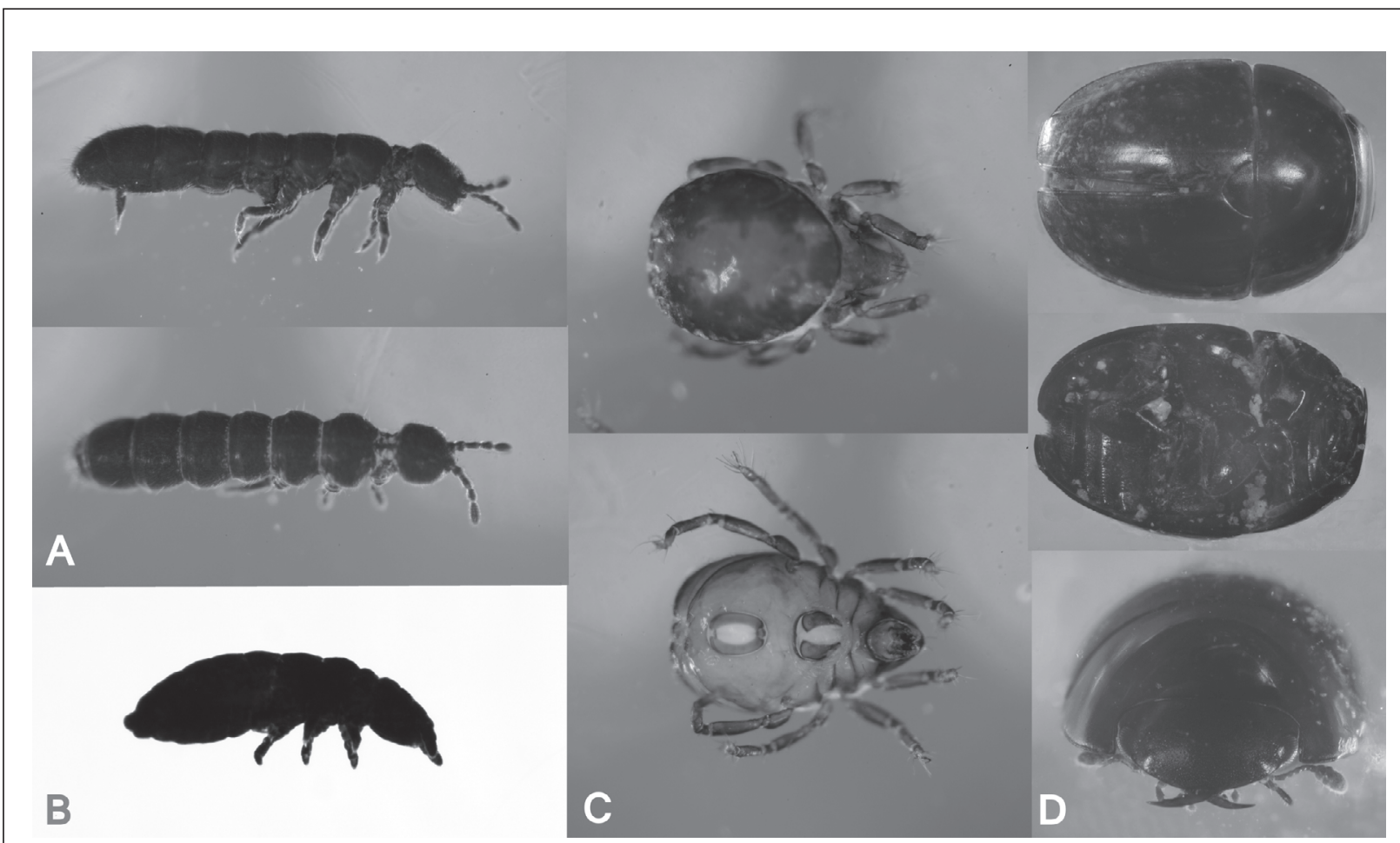

Fig. 5. Órdenes representados en los muestreos por embudo de Berlesse. A) Collembola (Cryptopygus antarcticus); B) Collembola (Gomphiocephalus hodgsoni); C) Acari; D) Coleoptera.

cocina. Sería importante determinar si se trata de un caso aislado o podemos estar ante una adaptación de T. maculipennis al ambiente antártico.

Por medio del embudo de Berlese se obtuvieron un total de 6749 individuos (Acari 55.96\%, Collembola 43.84\%, Coleoptera 0.02\% y Diptera 0.18\%) (Fig. 5). Por un lado, en la Isla Ardley el $81 \%$ de los ejemplares recolectados fueron ácaros, determinados a nivel familiar como Ameronothridae. La mayor proporción encontrada se explica por la asociación de estos arácnidos con los pingüinos que residen en esta zona protegida (Gressitt et al., 1963). Hasta el momento, Alaskozetes antarcticus intermedius es la especie reportada para el sitio y asociada a las colonias de pingüinos (Block \& Convey, 1995). Estudios futuros buscarán determinar los ejemplares para confirmar si se trata de la especie mencionada anteriormente.

Por otro lado, en los tanques de combustible en la Base de la Federación Rusa predominaron los colémbolos (99.8\%). Esto puede deberse a que este sitio presenta un rico tapiz vegetal que cubre grandes zonas del suelo. Se encontraron dos especies de colémbolos, Cryptopygus antarcticus Willem, 1901 y Gomphiocephalus hodgsoni Carpenter, 1908, siendo el primero el más abundante.

Por último, el hallazgo del orden Coleoptera en el Lago Uruguay representa el primer registro del orden para las Shettland del Sur. Estudios en desarrollo en conjunto con el Dr. Peter Convey (BAS Science strategy team) buscan identificar a nivel específico el ejemplar el coleóptero encontrado en las proximidades de la BCAA.

Contar con un registro de las especies de artrópodos presentes en la península Fildes como parte del ASPA, permite obtener una línea de base para evaluar futuras perturbaciones en los ambientes naturales. Dentro de las cuales se encuentra, el incremento de la actividad humana reportada en la última década por Frenot et al. (2005). Se espera que este trabajo constituya una línea de base para futuros estudios en artrópodos antárticos, tanto nativos como exóticos.

\section{AGRADECIMIENTOS}

Este trabajo forma parte del proyecto Composición y estructura de la Artrópodo-fauna en áreas cercanas a la Base Científica Antártica Artigas del Instituto Antártico Uruguayo (IAU). Agradecemos al IAU por la financiación, a Eduardo Juri y al Dr. Ernesto Brugnoli por su colaboración en el proyecto. Agradecemos a Gabriela Eguren y un revisor anónimo por sus valiosas sugerencias que contribuyeron a mejorar el manuscrito. 


\section{REFERENCIAS}

Barticevic, E. 2015. The life history of the Antarctic midge. Beyond the south, 2:19-22.

Block W. \& Convey P. 1995. The biology, life cycle and ecophysiology of the Antarctic mite Alaskozetes antarcticus. Journal of Zoology of London, 236: 431-449.

Convey P. \& Block W. 1996. Antarctic Diptera: ecology, physiology and distribution. European Journal of Entomology,93, 1-13.

Convey P.\& Block, W. 1996. Antarctic Diptera: ecology, physiology and distribution. European Journal of Entomology, 93: 1-13.

Convey P., Gibson J.A.E., Hillenbrand C.D., Hodgson D.A., Pugh P.J.A., Smellie J.L. \& Stevens M.I. 2008. Antarctic terrestrial life - challenging the history of the frozen continent? Biological Reviews, 83: 103-117.

Convey P. \& Stevens M.I. 2007. Antarctic biodiversity. Science, 317: 1877-1878.

Frenot Y., Chown S.L., Whinam J., Selkirk P.M., Convey P., Skotnicki M. \& Bergstrom, D.M. 2005. Biological invasions in the Antarctic: extent, impacts and implications. Biological Reviews, 80: 45-72.

Gañán Mora M., Contador T.A. \& Kennedy J.H. 2015. La vida en los extremos: el uso de SIG para estudiar la distribución de la mosca antártica alada, Parochlus steinenii (Diptera: Chironomidae), en las Islas Shetland del Sur (Antártica marítima). Análisis espacial y representación geográfica: innovación y aplicación ISBN: 978-84-92522-95-8

Gressitt J.L., Leech R.E. \& Wise K.A. J. 1963. Entomological Investigation in Antartica. Pacific Insects, 5(1): 287-304.

Hahn S. \& Reinhardt K. 2006. Habitat preference and reproductive traits in the Antarctic midge Parochlus steinenii (Diptera: Chironomidae). Antarctic Science, 18(2): 175-181.

Hughes K.A. \& Convey P. 2012. Determining the native/ non-native status of newly discovered terrestrial and freshwater species in Antarctica e Current knowledge, methodology and management action. Journal of Environmental Management, 93: 52-66.
Kennedy A.D. 1995. Antarctic terrestrial ecosystem response to global environmental change. Annual Review of Ecology and Systematics, 26: 683-704.

Kim J.H., Ahn I.Y., Lee K.S., Chung H. \& Choi H.G. 2007. Vegetation of Barton Peninsula in the neighbourhood of King Sejong Station (King George Island, maritime Antarctic). Polar Biology, 30: 903-916.

Longcore T. 2003. Terrestrial Arthropods as Indicators of Ecological Restoration Success in Coastal Sage Scrub (California, U.S.A.). Restoration Ecology, 11(4): 397-409.

Patrimonio Natural. 2009. Península Fildes ASPA 125. URL: http://www.patrimonionatural.com/HTML/ provincias/antartida/ASPA125Fildes/ ASPA125Fildes.asp

Rothwell D.R. 1990. The Antarctic Treaty System: Resource development, environmental protection or disintegration? Arctic, 43(3): 284291.

Seastedt T.R. \& Crossley Jr. D.A. 1984. The influence of arthropods on ecosystems. BioScience, 34(3): 157-161.

Serrano E. 2003. Paisaje Natural y Pisos Geoecológicos en las áreas libres de hielo de la Antártida marítima (Islas Shetland del Sur). Boletín de la A.G.E., 35: 5-32.

Tilbrook P.J. 1967. The Terrestrial Invertebrate Fauna of the Maritime Antarctic. Philosophical Transactions of the Royal Society of London. Series B Biological Sciences, 252(777): 261-278.

Uehara-Prado M., de Oliveira Fernandes J., de Moura Bello A., Machado G., Santos A.J., Zagury Vazde-Mello F. \& Lucci Freitas A.V. 2009. Selecting terrestrial arthropods as indicators of small-scale disturbance: A first approach in the Brazilian Atlantic Forest. Biological Conservation, 142: 1220-1228.

Volonterio O., Ponce de León R., Convey P., \& KrzemiD ska E. 2013. First record of Trichoceridae (Diptera) in the maritime Antarctic. Polar Biology, 36(8): 1125-1131.

Fecha de Recepción: 11 de Noviembre de 2017 Fecha de Aceptación: 2 de Julio de 2018 